\title{
Nearwork-Induced Transient Myopia in Preadolescent Hong Kong Chinese
}

\author{
James Stuart Wolffsohn, ${ }^{1}$ Bernard Gilmartin, ${ }^{1}$ Roger Wing-hong Li, ${ }^{2}$ \\ Marion Hastings Edwards, ${ }^{2}$ Sandy Wing-shan Chat, ${ }^{2}$ John Kwok-fai Lew, ${ }^{2}$ and \\ Bibianna Sin-ying $\mathrm{Yu}^{2}$
}

Purpose. To compare the magnitude and time course of nearwork-induced transient myopia (NITM) in preadolescent Hong Kong Chinese myopes and emmetropes.

Method. Forty-five Hong Kong Chinese children, 35 myopes and 10 emmetropes aged 6 to 12 years (median, 7.5), monocularly viewed a letter target through a Badal lens for 5 minutes at either 5.00- or 2.50-D accommodative demand, followed by 3 minutes of viewing the equivalent target at optical infinity. Accommodative responses were measured continuously with a modified, infrared, objective open-field autorefractor. Accommodative responses were also measured for a countercondition: viewing of a letter target for 5 minutes at optical infinity, followed by 3 minutes of viewing the target at a 5.00-D accommodative demand. The results were compared with tonic accommodation and both subject and family history of refractive error.

RESULTs. Retinal-blur-driven NITM was significantly greater in Hong Kong Chinese children with myopic vision than in the emmetropes after both near tasks, but showed no significant dose effect. The NITM was still evident 3 minutes after viewing the 5.00-D near task for 5 minutes. The magnitude of NITM correlated with the accommodative drift after viewing a distant target for more than 4 minutes, but was unrelated to the subjects' or family history of refractive error.

Conclusions. In a preadolescent ethnic population with known predisposition to myopia, there is a significant posttask blurdriven accommodative NITM, which is sustained for longer than has previously been found in white adults. (Invest Ophthalmol Vis Sci. 2003;44:2284-2289) DOI:10.1167/iovs.02-0373

$\mathrm{T}$ he association between the onset and development of myopia and sustained nearwork has figured in the research literature for many years ${ }^{1}$ and has instigated a variety of experimental protocols to compare pre- and posttask measures of oculomotor function. Of particular interest has been the finding that immediately after a period of sustained nearwork, myopes are less able to accommodate accurately for distance

From the ${ }^{1}$ Neurosciences Research Institute, School of Life and Health Sciences, Aston University, Birmingham, United Kingdom; and the ${ }^{2}$ Centre for Myopia Research, Faculty of Health and Social Sciences, The Hong Kong Polytechnic University, Hong Kong, China.

Supported by Grant A436 from the Centre for Myopia Research, Area of Strategic Development, The Hong Kong Polytechnic University. Johnson \& Johnson (Hong Kong) Ltd. kindly provided disposable contact lenses.

Submitted for publication April 15, 2002; revised October 4 and December 17, 2002; accepted January 16, 2003.

Commercial relationships policy: $\mathrm{N}$.

The publication costs of this article were defrayed in part by page charge payment. This article must therefore be marked "advertisement" in accordance with 18 U.S.C. $\$ 1734$ solely to indicate this fact.

Corresponding author: James S. Wolffsohn, Neurosciences Research Institute, School of Life and Health Sciences, Aston University, Birmingham B4 7ET, UK; j.s.w.wolffsohn@aston.ac.uk. than emmetropes or hyperopes, a phenomenon described as nearwork-induced transient myopia (NITM). ${ }^{2}$ Ciuffreda and Wallis ${ }^{2}$ found that the initial accommodative inaccuracy averaged approximately $0.35 \mathrm{D}$ overaccommodation in both their early-onset (EOMs, $n=13$ ) and late-onset (LOMs, $n=11$ ) myopic groups. Neither the emmetropic group $(n=11)$ nor the hyperopic group $(n=9)$ exhibited significant NITM. The myopic groups, however, were distinguished by differences in the time taken subsequently to reach a stable optimum level of accommodation for distance vision. LOMs were found to take almost twice as long to reach optimum distance accommodation levels than EOMs (i.e., 63 seconds versus 35 seconds), and the authors proposed that the consequent retinal defocus and degradation in retinal image contrast were sufficient to trigger compensatory blur-driven growth of the posterior vitreous chamber. The proposal is supported by a body of work on myopia in animals that indicates that even modest levels of retinal defocus, encompassed by depth of focus, can be sufficient to induce ocular growth. ${ }^{3}$

Both myopic groups in the study by Ciuffreda and Wallis ${ }^{2}$ were drawn from a sample of adult optometry students with a mean age of approximately 24 years. The authors suggested that the continuing propensity to NITM shown in these individuals represents an inherent characteristic that, earlier in life, induces temporary myopia after exposure to sustained nearwork. In EOM the exposure produces derailment of a preprogrammed pattern of ocular growth in the developing eye. In LOM, where ocular growth due to emmetropization has slowed or ceased before the onset of myopia, ${ }^{4,5}$ the derailment is more directly attributable to nearwork exposure. Ciuffreda and Wallis ${ }^{2}$ showed clearly that white adults with early- or late-onset myopia, measured under binocular, free-space, natural viewing conditions, were susceptible to accommodative nearwork aftereffects, even in the presence of normal-distance blur-related feedback. ${ }^{2}$

Of special relevance to the present study is the subsequent report by Ciuffreda and Thunyalukul ${ }^{6}$ on a group of children aged 4.7 to 9.9 years. The study demonstrated that juvenile myopes $(n=10)$ were more susceptible to NITM than emmetropes $(n=10)$ and further, that subjects in each refractive group who exhibited an initial NITM, sustained the effect throughout a 2-minute posttask period. That NITM is more sustained in children than in adults was attributed by the authors to differences in sympathetic innervation and/or sensitivity to blur. It was further postulated that NITM in conjunction with the decreased blur-driven accommodative response at near associated with newly developed myopia in children may lead to a time-integrated cumulative retinal defocus that triggers axial elongation.

The observation that the ability to detect blur is reduced in adults with myopia ${ }^{7}$ has been examined recently in a cohort of 20 children (aged 8-12 years), with or without myopic vision, ${ }^{8}$ for two different black and white targets (text and scenes) and illumination conditions. There was no correlation between blur thresholds and refractive error magnitude, refractive error progression (over the previous year), or contrast sensitivity. 
However, blur-detection ability showed significantly greater individual variability in juvenile myopes, which led the authors to suggest that subgroups may differ in ability to detect blur.

Further consideration of the role of retinal defocus is opportune after the recent important advances in theoretical modeling of the development of refractive error. ${ }^{9-12}$ The Incremental Retinal Defocus Theory proposed by Hung and Ciuffreda ${ }^{11}$ offers significant insight into the myopigenic nature of retinal defocus. The critical element of the theory, as it relates to nearwork, is that the detection mechanism triggering ocular growth does not depend on the sign of the retinal blur, but rather on the change in magnitude of blur during genetically programmed ocular growth-the rate of ocular growth being dependent on the change in magnitude of retinal defocus, regardless of how it is generated.

A compelling research question is how processing of retinal defocus amalgamates with mechanisms of accommodative adaptation and its neuropharmacologic control. Regarding the former, a computer simulation model constructed by Hung and Ciuffreda ${ }^{13}$ extended a previous adaptation model ${ }^{14}$ by adding a proximal component, ${ }^{15}$ to provide a more realistic and comprehensive representation of the accommodation system and to extend the model to NITM data. The adaptive gain $\left(K_{\mathrm{A}}\right)$ was used to modify the time constant of the controller and variations in $K_{\mathrm{A}}$ (using the original Ciuffreda and Wallis data set ${ }^{2}$ ) were shown to be the principal determinant of variations in NITM between refractive groups. Indeed, differences in accommodative gain between refractive groups were thought by Culhane and Winn ${ }^{16}$ to account for their finding, using closedloop dynamic accommodation measures, that late-onset young adult myopes have significantly longer reflex near-far response times after sustained 3-minute near tasks.

In a recent study, using similar investigatory techniques, Winn et al. ${ }^{17}$ present evidence to support proposals by several groups (see review by Gilmartin ${ }^{18}$ ) that autonomic neuropharmacologic modulation of accommodative adaptation and NITM is a feasible putative control system for sustained near vision. The principal systems concern the integration of parasympathetic and sympathetic innervation of ciliary smooth muscle, such that sympathetic inhibition (through $\beta 2$-adrenoceptors) is augmented by concurrent parasympathetic activity (through M3 cholinergic receptors). An anomaly in integration, say for example by a deficit in inhibition, may thus predispose an individual to abnormal postnearwork accommodative responses and possibly have etiological significance in the development of myopia, although only approximately $30 \%$ of individuals appear to have access to a sympathetic facility. ${ }^{19}$ Winn et al. ${ }^{17}$ used topical $\beta$-adrenoceptor antagonist drugs in five young adult emmetropes to demonstrate inhibitory sympathetic modulation of accommodation responses to dark room open-loop or closed-loop conditions involving systematic stepwise and sinusoidal changes in stimulus vergence.

Whereas the nature of autonomic control and sustained near vision has been addressed in several studies in young adults ${ }^{18}$ very few have involved children. In this regard, the recent preliminary report by Chen et al. ${ }^{20}$ is of particular relevance to the present study, as its purpose was to investigate, in Hong Kong children (aged 8-12 years), the effect of a topical $\beta$ antagonist drug, timolol maleate $(0.5 \%, 20 \mu \mathrm{L})$, on tonic accommodation measures, before and after playing a 5 -minute video game. The profile of posttask responses in the 30 children (10 emmetropes and 20 myopes, 5 with stable and 15 with progressing myopia) appeared to match those shown in previous studies on young adults. The results showed that, whereas timolol increased the magnitude of adaptation in stable myopes, it had no effect on adaptation in progressing myopia and had a counteradaptive effect in emmetropes. The absence of effect of timolol on adaptation in children with progressive myopia implies that they have a deficit in sympathetic inhibition.

The composite near response epitomizes the nature of autonomic control. That is, a profound mediation of central and peripheral processes to ensure an optimum balance between internal and external demands on the organism and future work is needed to elucidate further whether sympathetic control pervades the near vision complex as a whole or is restricted to specific elements of the response.

The purpose of this study was to determine the magnitude and time course of NITM induced solely by retinal blur with a variety of stimulus demands in preadolescent Hong Kong Chinese, a group that is particularly susceptible to environmental myopia and that is in the developing phase of myopia. ${ }^{21-23}$ Monocular viewing through a Badal optical system was used to examine the steady-steady blur-driven component of accommodation and to allow control of proximal accommodation.

\section{MethodS}

Forty-five Hong Kong Chinese children aged 6 to 12 years participated in the study. Thirty-five myopes ( 21 boys, 14 girls) of median age 8.0 years (range, 6-12), mean spherical equivalent refractive error (MSE) $-3.41 \pm 0.99 \mathrm{D}$ (range, -1.63 to $-5.63 \mathrm{D}$ ), were recruited. All were habitual single-vision spectacle wearers, but had refraction fully corrected with hydrophilic daily disposable contact lenses for the purposes of this experiment (2-hydroxyethyl methacrylate [HEMA] lenses, $58 \%$ water content; Acuvue Dailies, Vistakon, Johnson \& Johnson Vision Care, Jacksonville, FL). Owing to the high prevalence of myopia in Hong Kong Chinese children older than 8 years, the control emmetropic sample was smaller in number (five boys, five girls; MSE $+0.00 \pm 0.23 \mathrm{D}$; range, +0.50 to $-0.25 \mathrm{D})$ and slightly younger (median age, 7.0 years; range, 6-10) than the myopic group. Subjects were not taking any medication and were prescreened to ensure that they had no ocular disease or binocular vision abnormalities. Corrected visual acuity ranged from -0.04 to $0.02 \mathrm{log}$ minimum angle of resolution (logMAR; average, $0.00 \pm 0.01$ ), and astigmatism was less than 1.25 D. Informed consent was obtained from a subject's parent after explanation of the nature and possible consequences of the study. The research adhered to the tenets of the Declaration of Helsinki and was approved by institutional human experimentation committee of The Hong Kong Polytechnic University.

The infra-red (IR) autorefractor (SRW-5000; Shin-Nippon Ophthalmic Instruments, Tokyo, Japan) was used to measure the accommodative state of the subject's right eye throughout the experimental trials. The instrument provides an open field of view and quantifies accommodation by image analysis of an IR ring of light, reflected from the retina. Previous studies have shown that this system provides measures of high validity and repeatability in both adult ${ }^{24}$ and juvenile ${ }^{25}$ myopes. The autorefractor was modified to allow continuous recording of accommodation, with a resolution of less than $0.01 \mathrm{D} .{ }^{26}$ Pupil size was always greater than $3 \mathrm{~mm}$, resulting in an approximately constant depth of focus. ${ }^{27}$

The visual axis of the infrared autorefractor was aligned with the right eye, with the left eye occluded. The subject viewed a row of Arabic letters ( $>90 \%$ contrast), subtending 1 minute of arc (equivalent to $0.00 \operatorname{logMAR}$ ), and was prompted at regular intervals to maintain fixation, focus, and attention on the letters. The letters were viewed along the visual axis through a +5.00-D Badal system, and the accommodative demand was changed instantaneously (i.e., in $<100 \mathrm{~ms}$ ) using a solenoid stepper motor. The Badal system ensured the same size and contrast of the target, regardless of the accommodative demand. The subject initially viewed the letter target, of luminance $20.0 \mathrm{~cd} / \mathrm{m}^{2}$, located $20 \mathrm{~cm}$ behind the Badal lens (i.e., imaged at optical infinity), and concurrent measures of the autorefractor IR measurement ring were recorded dynamically, using a computer program (Labview; National Instruments, Austin, TX), and statically, using the autorefractor's preset internal calibration. All data are presented in relation to the subject's baseline distance 

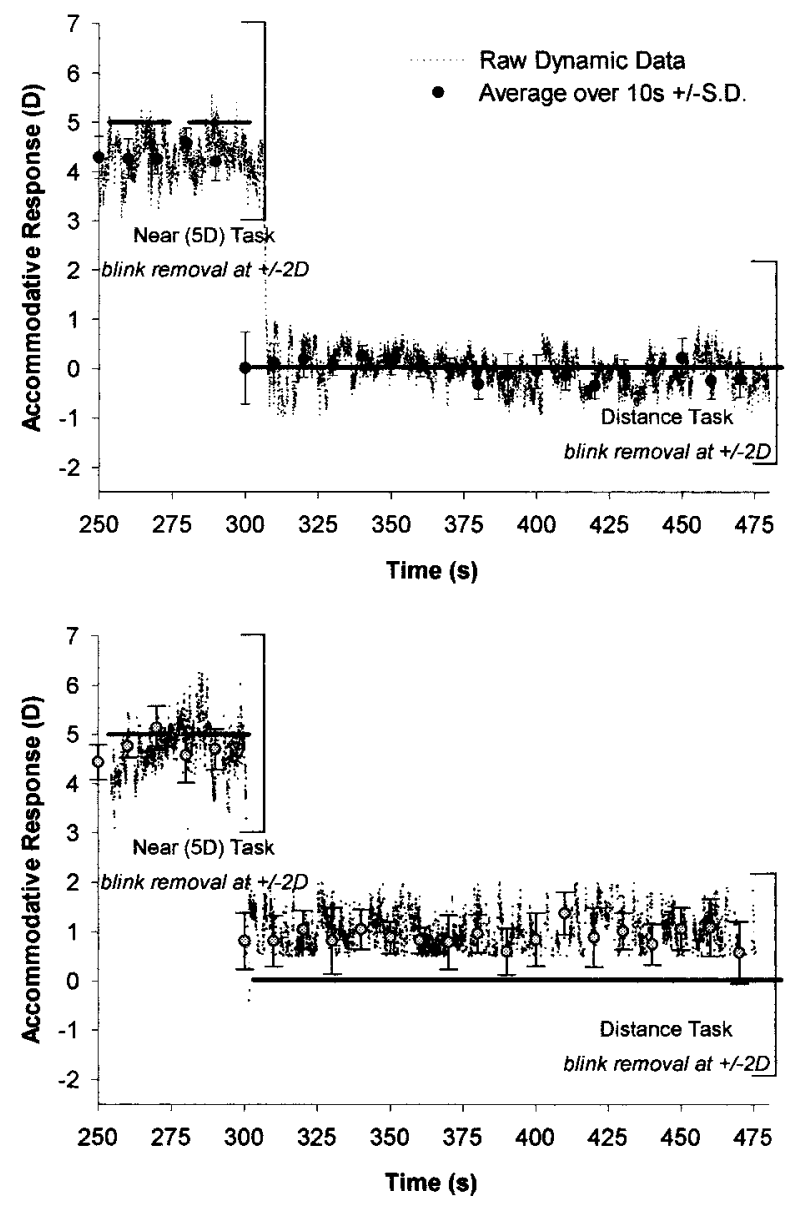

FigURE 1. Example of typical data from an emmetrope (top trace) and a myope (bottom trace) viewing a letter target at 5.0-D accommodative demand for 5 minutes, followed by optical infinity for 3 minutes. The figure demonstrates the data analysis performed on the dynamic accommodative trace

accommodative state $(0.0 \mathrm{D})$. The lead of accommodation, indicated by the static autorefractor readings when the subject viewed the distant target, is shown on the $y$ axis of Figures 2-4.

Initially, accommodation was monitored dynamically while the subject viewed a $0.1 \mathrm{cyc} / \mathrm{deg}$ difference-of-gaussian target (DoG) at 25 $\mathrm{cd} / \mathrm{m}^{2}$ for 5 minutes placed at optical infinity to determine the tonic accommodative level. ${ }^{28}$ Three trials were randomly allocated to each subject, with the test letters viewed for 5 minutes at one distance, after which the accommodative demand of the target was instantaneously changed to its new value and the target viewed for a further 3 minutes: a near task at $5.0 \mathrm{D}$, followed by 3 minutes at $0.0 \mathrm{D}$; a near task at 2.5 $\mathrm{D}$, followed by 3 minutes at $0.0 \mathrm{D}$; and a distance task at $0.0 \mathrm{D}$, followed by 3 minutes at $5.0 \mathrm{D}$. The online analysis system monitored both the position of the target and the corresponding accommodative response, facilitating assessment of response latency characteristics. Demographic data and details of family history of refractive error were also collected for each subject.

Split-plot analysis of variance (StatView; SAS, Cary, NC) ${ }^{29}$ was used to examine the differences in posttask NITM between the refractive groups over time. Pearson's product moment coefficient and stepwise analysis were used to test the relationship between NITM, demographic data, and family history.

\section{RESUlts}

Figure 1 shows an example of the raw data from a typical myope (bottom trace) and a typical emmetrope (top trace).
Blink artifacts were removed from the dynamic accommodative trace by removing data that were not within $2 \mathrm{D}$ of the task demand. To allow comparison with previous research, the trace was averaged into 10 -second intervals and the standard deviation calculated (from approximately 350 dynamic readings). Because the accommodative response is usually complete in less than 1 second $^{30}$ and the slow phase accommodative response is the principal component in NITM, ${ }^{12}$ the first second of data after the stimulus changed distance was removed from the analysis.

NITM, over the 3-minute posttask interval, was significantly greater for each averaged interval in Hong Kong Chinese juvenile myopes than in emmetropes $(\mathrm{F}=11.40, P<0.001$; Fig. 2$)$. NITM in myopes was significantly greater than the baseline accommodative response for a distant target over the entire 3-minute posttask period (Table 1). There was no significant NITM in accommodation after the near task in emmetropes (Table 1). There was no significant reduction in NITM over the 3-minute posttask time interval after the 5.0- and 2.5-D task demand (split-plot ANOVA, F $=1.10, P=0.30$; Fig. 3). There was also no interaction between task demand, time after near task, and refractive status (i.e., myopia versus emmetropia; $P>$ $0.50)$.

When the task condition was reversed so that subjects viewed a distant target at $0.0 \mathrm{D}$ accommodative demand for 5 minutes, followed by a near target at $5.0 \mathrm{D}$ (Fig. 4), emmetropes were better able to maintain their baseline accommodative response to the target at optical infinity than myopes (Table 1). However, the lag for myopes was still smaller than the magnitude of NITM after a near task. There was no significant difference between myopes and emmetropes in their accommodative response to the subsequent near task compared with baseline (unpaired $t$-test: $P=0.43$ ). Therefore, the accommodative response domain (i.e., the difference between mean accommodative response for distance and near task) was significantly smaller for myopes than for emmetropes in all three task conditions, but more so when a near target was viewed before rather than after the distant target (Table 2).

The mean tonic accommodative level to the DoG target was $+0.82 \pm 0.75 \mathrm{D}$ in juvenile myopes and $+0.93 \pm 0.56 \mathrm{D}$ in emmetropes. The age of onset of myopia ranged from 6 to 10 years (mean, $7.7 \pm 1.1$ ). The mean reported parental refractive error for the myopic group was $-2.87 \pm 2.80 \mathrm{D}$, with $29 \%$ emmetropic. The refractive error of $13 \%$ of grandparents was not known, but of the remainder, the reported MSE was $-0.43 \pm 1.71 \mathrm{D}$, with $82 \%$ emmetropes.

The relationships between the magnitude of NITM in myopic Hong Kong Chinese children and tonic accommodative

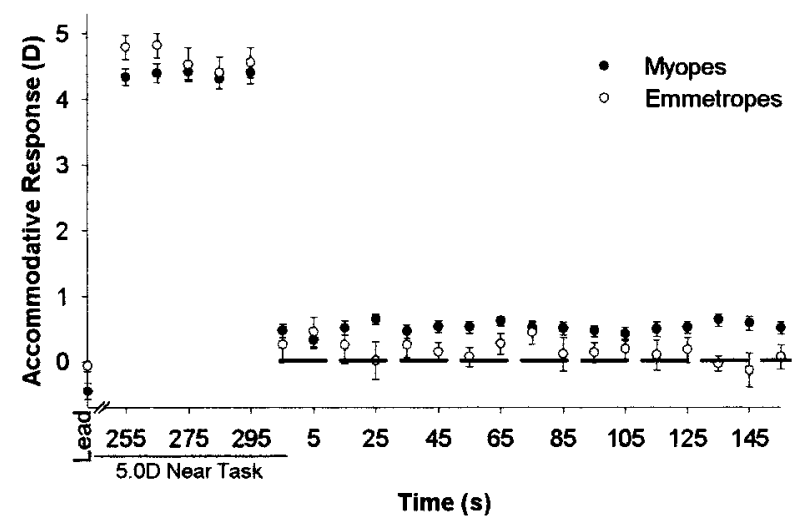

FIGURE 2. Accommodative response to viewing a near stimulus at 5.0-D accommodative demand for 5 minutes, followed by $0.0 \mathrm{D}$ for 3 minutes. Dasbed line: baseline accommodation to a distant target. $n=$ 35 myopes, 10 emmetropes. Error bars, 1 SEM. 
TABle 1. Mean Accommodative Level of Myopic and Emmetropic Groups while Performing the Tasks

\begin{tabular}{|c|c|c|c|c|}
\hline & \multirow{2}{*}{$\frac{\text { Prechange }}{250-300 \mathrm{sec}}$} & \multicolumn{3}{|c|}{ Postchange } \\
\hline & & $1-60 \mathrm{sec}$ & $61-120 \mathrm{sec}$ & 121-180 sec \\
\hline \multicolumn{5}{|l|}{ Myopes } \\
\hline 5.0 to $0.0 \mathrm{D}$ & $4.35 \pm 0.63$ & $0.47 \pm 0.46 \dagger$ & $0.49 \pm 0.43 \dagger$ & $0.52 \pm 0.44 \dagger$ \\
\hline 2.5 to $0.0 \mathrm{D}$ & $1.87 \pm 0.51$ & $0.39 \pm 0.43 \dagger$ & $0.44 \pm 0.46 \dagger$ & $0.37 \pm 0.56 \dagger$ \\
\hline 0.0 to $5.0 \mathrm{D}$ & $0.30 \pm 0.56^{*}$ & $4.42 \pm 0.67$ & $4.38 \pm 0.66$ & $4.34 \pm 0.66$ \\
\hline \multicolumn{5}{|l|}{ Emmetropes } \\
\hline 5.0 to $0.0 \mathrm{D}$ & $4.62 \pm 0.43$ & $0.19 \pm 0.58$ & $0.20 \pm 0.43$ & $0.10 \pm 0.45$ \\
\hline 2.5 to $0.0 \mathrm{D}$ & $1.96 \pm 0.65$ & $0.12 \pm 0.42$ & $0.03 \pm 0.34$ & $0.03 \pm 0.53$ \\
\hline 0.0 to $5.0 \mathrm{D}$ & $0.06 \pm 0.60$ & $4.44 \pm 0.38$ & $4.40 \pm 0.26$ & $4.42 \pm 0.40$ \\
\hline
\end{tabular}

Data are the mean \pm SD. Significance tested for distance viewing with respect to the baseline accommodative response.

$* P<0.01$.

$\dagger P<0.001$.

level, age-of-onset and magnitude of myopia, magnitude of parent and grandparent refractive error, and the drift in baseline accommodative level 50 seconds before the change in accommodative demand (i.e., between 250 and 300 seconds) were examined. Best subset regression analysis showed that $30.2 \%$ of the variance in the magnitude of NITM could be accounted for by a combination of all these features. However, most variance was accounted for by the drift in baseline accommodative level (24.2\%), followed by the age of onset of myopia (5.3\%).

\section{Discussion}

The study shows that preadolescent myopic Hong Kong Chinese children are significantly more susceptible than their emmetrope peers to blur-driven NITM induced by sustained near vision. The magnitude of NITM found in the juvenile myopes was of an order similar to that reported by Ciuffreda and Wallis ${ }^{2}$ for white adults with early-onset myopia under binocular open-view natural viewing conditions. A distinguishing feature of NITM in the present study is, however, its persistence and lack of dependence on the level of accommodative demand of the near task. Regarding the latter and consistent with a number of previous studies, ${ }^{31,32}$ myopes also exhibited significantly greater lags of accommodation at near than emmetropes and hence the composite profile of accommodative response, expressed herein as accommodative re-

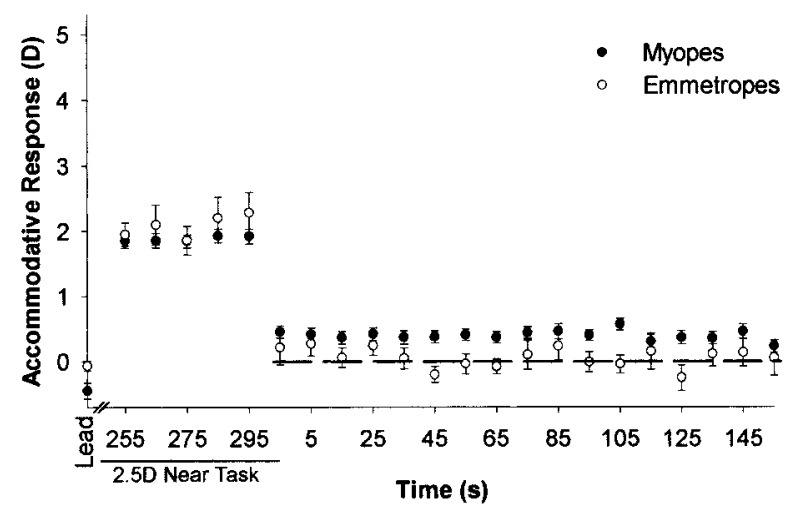

Figure 3. Accommodative response to viewing a near stimulus at 2.5-D accommodative demand for 5 minutes, followed by $0.0 \mathrm{D}$ for 3 minutes. Dashed line: baseline accommodation to a distant target. $n=$ 35 myopes, 10 emmetropes. Error bars, 1 SEM. sponse domain, is shown to be significantly reduced in myopes compared with emmetropes.

There is also substantial evidence for an association between the onset of myopia and a decreased accommodative response to retinal blur, ${ }^{7,8,33}$ an effect which, by inducing regression of accommodation to an intermediate location, ${ }^{1}$ may in part account for the significantly reduced accommodative domain found in the juvenile myopes used in this study. In their comprehensive monograph on accommodation and myopia, Ong and Ciuffreda ${ }^{34}$ identify retinal blur as the principal component of NITM and consider its putative etiological role in myopia. The mechanisms proposed in later work on NITM by Ciuffreda and Wallis ${ }^{35}$ may be especially relevant to the juvenile myopes used in this study, as, given that it is a group characterized by rapidly progressing myopia and associated increase in lag of accommodation at near, it may be particularly susceptible to the time-integrated retinal defocus for both near and distance hypothesized by the authors. Both sources of retinal blur have potential myopigenic effects, owing to the authors' further proposition that small amounts of retinal defocus emanating from accommodative dysfunction in the myopic eye, but not exceeding the eye's depth of focus, may not be sufficient to provide directional information, as blur defocus has no directional clues. ${ }^{36}$ The contribution of retinal blur to reflex accommodation is equivocal, however. For example, Kruger et al. ${ }^{37}$ have shown, measuring accommodation continuously under open-loop conditions, that both achromatic

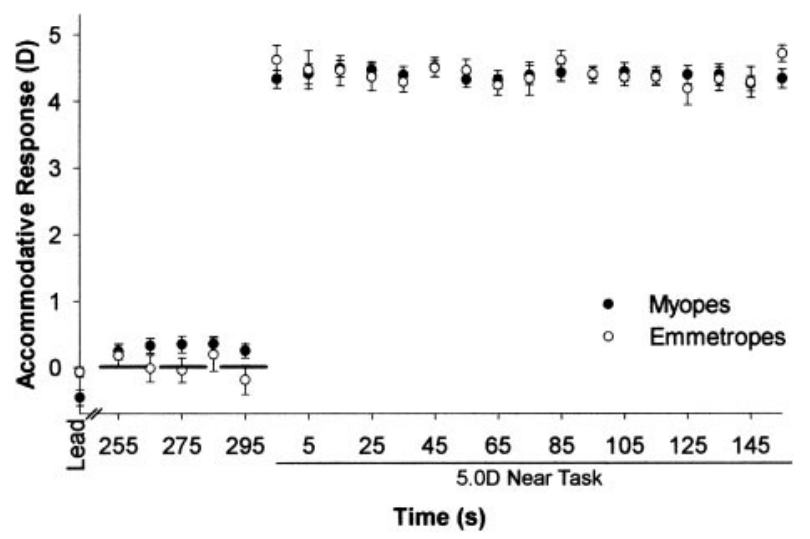

Figure 4. Accommodative response to viewing a distant stimulus at $0.0 \mathrm{D}$ accommodative demand for 5 minutes, followed by a near task of 5.0 D accommodative demand for 3 minutes. Dashed line: baseline accommodation to a distant target. $n=35$ myopes, 10 emmetropes. Error bars, 1 SEM. 
TABle 2. Accommodative Domain for the Tasks in the Study

\begin{tabular}{cccc}
\hline Task & Myopes & Emmetropes & Significance \\
\hline 5.0 to $0.0 \mathrm{D}$ & $3.86 \pm 0.70$ & $4.46 \pm 0.63$ & 0.02 \\
2.5 to $0.0 \mathrm{D}$ & $1.47 \pm 0.64$ & $1.90 \pm 0.63$ & 0.08 \\
0.0 to $5.0 \mathrm{D}$ & $4.08 \pm 0.70$ & $4.36 \pm 0.67$ & 0.28 \\
\hline
\end{tabular}

Accommodative domain is the difference between mean accommodative responses. Data are the mean \pm SD.

and chromatic directional stimuli specify ocular focus and drive reflex accommodation. The inability to demonstrate a significant dose effect in this study may be attributable to accommodative adaptation processes that attain, with sustained near fixation, a subthreshold level of retinal blur that is independent of the magnitude of accommodative stimulus but sufficient to cause NITM.

Whereas the substantial and persistent NITM induced by a relatively modest exposure to sustained near vision may be evidence that nearwork constitutes a potent environmental trigger for progression of myopia in the myopic eye of Hong Kong Chinese, of interest is whether the absence of NITM found in emmetropes precludes the role of NITM as a putative precursor to development of myopia.

In this context, a longitudinal study of propensity to NITM in the Hong Kong Chinese eye and its role in the genesis of myopia would be especially valuable, as epidemiologic studies show that the vision in at least $70 \%$ of the juvenile emmetropes used in this study will become significantly myopic over the next few years. ${ }^{21}$ Further, a parallel study in eyes of whites may elucidate whether pronounced NITM contributes to the higher degree and prevalence of myopia in Chinese populations. ${ }^{38}$

Such studies are likely, however, to present methodological difficulties, owing to the rapid progression of myopia evident in Hong Kong Chinese (MSE 1.47 $\pm 0.74 \mathrm{D}$ per year for the sample used in this study). For this reason perhaps, neither the degree of myopia nor the family history of myopia showed a significant correlation with the magnitude of NITM found in the juvenile myopes. Of further interest for future work is the nature of NITM in hyperopia, a group rarely included in study samples but one that could be crucial to our full understanding of the etiology of myopia. Recently Ciuffreda and Lee ${ }^{39}$ have shown that not only are hyperopes markedly resistant to NITM but that they can exhibit substantial and sustained posttask hyperopic shifts of the order of $-0.6 \mathrm{D}$ after a continuous 4-hour task at their habitual reading distance.

The family history of refractive error reported in this study confirms prevalence levels previously published for corresponding generations ${ }^{40,41}$ and highlights the rapidly increasing prevalence of myopia in Hong Kong Chinese. ${ }^{21,22}$ The association between accommodation, nearwork, and the onset and development of myopia has figured in the literature over many years, ${ }^{1}$ and, more recently, nearwork has been proposed as a significant environmental influence in genetic and familial models of development of refractive error. ${ }^{42-44}$ We present evidence that propensity to substantial and sustained blurdriven NITM after nearwork is a feature of the young Hong Kong Chinese myopic eye and propose that this may exacerbate genetic predisposition of these eyes to progression of myopia.

\section{Acknowledgments}

The authors thank Richard Armstrong for statistical advice.

\section{References}

1. Rosenfield M. Accommodation and myopia. In: Rosenfield M, Gilmartin B, eds. Myopia and Nearwork. London: Butterworth Heinemann; 1998:91-116.

2. Ciuffreda KJ, Wallis DM. Myopes show increased susceptibility to nearwork aftereffects. Invest Ophthalmol Vis Sci. 1998;39:17971803.

3. Smith EL. Environmentally induced refractive errors in animals. In: Rosenfield M, Gilmartin B, eds. Myopia and Nearwork. London: Butterworth Heinemann; 1998:57-90.

4. Sorsby A, Benjamin B, Sheridan M. Refraction and its components during the growth of the eye from the age of three. Medical Research Council Special Report Series. No. 301: London: HMSO; 1961.

5. Larsen JS. The sagittal growth of the eye. IV. Ultrasonic measurement of the depth of the anterior chamber from birth to puberty. Acta Ophthalmol. 1971;49:873-886.

6. Ciuffreda KJ Thunyalukul V. Myopic nearwork aftereffects in children [ARVO Abstract]. Invest Ophthalmol Vis Sci. 1999;40:S448. Abstract nr 2365.

7. Rosenfield M, Abraham-Cohen JA. Blur sensitivity in myopes. $O p$ tom Vis Sci. 1999;76:303-307.

8. Schmid KL, Iskander DR, Li WH, Edwards MH, Lew JKF. Blur detection thresholds in childhood myopia: single and dual target presentation. Vision Res. 2002;42:239-247.

9. Flitcroft DI. A model of the contribution of oculomotor and optical factors to emmetropization and myopia. Vision Res. 1998;38: 2869-2879.

10. Hung GK, Ciuffreda KJ. Model of refractive error development. Curr Eye Res. 1999;19:41-42.

11. Hung GK, Ciuffreda KJ. A unifying theory of refractive error development. Bull Math Biol. 2000;62:1087-1108.

12. Hung GK, Ciuffreda KJ. Models of refractive error development. In: Hung GK, Ciuffreda KJ, eds. Models of the Visual System. New York: Kluwer Academic/Plenum Press; 2002;643-677.

13. Hung GK. Adaptation model of accommodation and vergence. Ophthalmic Physiol Opt. 1999;12:319-326.

14. Hung GK, Ciuffreda KJ. Adaptation model of nearwork-induced transient myopia. Ophthalmic Physiol Opt. 1999;19:151-158.

15. Hung GK, Ciuffreda KJ, Rosenfield M. Proximal contribution to a linear static model of accommodation and vergence. Ophthalmic Physiol Opt. 1996;16:34-41.

16. Culhane HM, Winn B. Dynamic accommodation and myopia. Invest Ophthalmol Vis Sci. 1999;40:1968-1974.

17. Winn B, Culhane HM, Gilmartin B, Strang NC. Effect of beta adrenoceptor antagonists on autonomic control of ciliary smooth muscle. Ophthalmic Physiol Opt. 2002;22:359-365.

18. Gilmartin B. Autonomic correlates of near-vision in emmetropia and myopia. In: Rosenfield M, Gilmartin B, eds. Myopia and Nearwork. London: Butterworth Heinemann; 1998:117-146.

19. Gilmartin B, Mallen EAH, Wolffsohn JS. Sympathetic control of accommodation: evidence for inter-subject variation. Ophthalmic Physiol Opt. 2002;22:366-371.

20. Chen JC, Schmid KL, Brown B, Edwards MH, Lew JKF, Yu BSY. The effect of timolol induced beta-adrenergic antagonism on accommodative adaptation in Hong Kong Chinese [ARVO Abstract]. Invest Ophthalmol Vis Sci. 2002;43:1513.

21. Edwards MH. The development of myopia in Hong Kong children between the ages of 7 and 12 years: a five-year longitudinal study. Ophthalmic Physiol Opt. 1999;19:286-294.

22. Lam CSY, Edwards M, Millodot M, Goh WSH. A 2-year longitudinal study of myopia progression and optical component changes among Hong Kong schoolchildren. Optom Vis Sci. 1999;76:370380.

23. Wu MMM, Edwards MH. The effect of having myopic parents: an analysis of myopia in three generations. Optom Vis Sci. 1999;76: 387-392.

24. Mallen EAH, Wolffsohn JS, Gilmartin B, Tsujimura S. Clinical evaluation of the Shin-Nippon SRW-5000 autorefractor in adults. Ophthalmic Physiol Opt. 2001;21:101-107. 
25. Chat SWS, Edwards MH. Clinical evaluation of the Shin-Nippon SRW-5000 autorefractor in children. Opbthalmic Pbysiol Opt. 2001;21:87-100.

26. Wolffsohn JS, Gilmartin B, Mallen EAH, Tsujimura S. Continuous recording of accommodation and pupil size using the Shin-Nippon SRW-5000 autorefractor. Ophthalmic Pbysiol Opt. 2001;21:108113.

27. Charman WN, Whitefoot H. Pupil diameter and the depth-of-field of the human eye as measured by laser speckle. Opt Acta. 1977; 24:1211-1216.

28. Rosenfield M, Ciuffreda KJ, Hung GK, Gilmartin B. Tonic accommodation: a review 1. Basic aspects. Ophthalmic Physiol Opt. 1993;13:266-284.

29. Snedecor GW, Cochran WG. Statistical methods. 7 th ed. Ames, IA: The Iowa State University Press; 1980:369-375.

30. Phillips S, Shirachi D, Stark L. Analysis of accommodation response times using histogram information. Am J Optom. 1972;49:389401.

31. Gwiazda J, Thorn F, Bauer J, Held R. Myopic children show insufficient response to blur. Invest Ophthalmol Vis Sci. 1993;34: $640-694$.

32. Jiang BC. Integration of a sensory component into the accommodation model reveals differences between emmetropia and lateonset myopia. Invest Ophthalmol Vis Sci. 1997;38:1511-1516.

33. Gwiazda J, Bauer J, Thorn F, Held R. A dynamic relationship between myopia and blur-driven accommodation in school-aged children. Vision Res. 1995;35:1299-1304.

34. Ong E, Ciuffreda KJ. Accommodation, Nearwork and Myopia.
Santa Ana, CA: Optometric Extension Program Foundation Press; 1997.

35. Abbott ML, Schmid KL, Strang NC. Differences in the accommodation stimulus response curves in adult myopes and emmetropes. Opbthalmic Physiol Opt. 1998;18:13-20.

36. Stark L. Neurological Control Systems. New York: Plenum; 1968.

37. Kruger PB, Mathews S, Katz M, Aggarwala KR, Nowbotsing S. Accommodation without feedback suggests directional signals specify ocular focus. Vision Res. 1997;37:2511-2526.

38. Wong TY, Foster PJ, Hee J, et al. Prevalence and risk factors for refractive errors in adult Chinese in Singapore. Invest Ophthalmol Vis Sci. 2000;41:2486-2494.

39. Ciuffreda KJ, Lee M. Differential refractive susceptibility to sustained near work. Ophthalmic Physiol Opt. 2002;22:372-384.

40. Goh WSH, Lam CSY. Changes in refractive trends and optical components of Hong Kong Chinese ages 19-39 years. Opbthalmic Pbysiol Opt. 1994;14:378-382.

41. Lam CSY, Goh WSH, Tang YK, et al. Changes in refractive trends and optical components of Hong Kong Chinese aged over 40 years. Ophthalmic Physiol Opt. 1994;14:383-387.

42. Lyhne N, Sjolie AK, Kyvik KO, Green A. The importance of genes and environment for ocular refraction and its determiners: a population based study among 20-45 year old twins. Br J Ophthalmol. 2001;85:1470-1476.

43. Saw S-M, Hong C-Y, Chia K-S, Stone RA, Tan D. Nearwork and myopia in young children. Lancet. 2001;357:390.

44. Hammond CJ, Snieder H, Gilbert CE, Spector TD. Genes and environment in refractive error: the twin eye study. Invest $O p b$ thalmol Vis Sci. 2001;42:1232-1236. 